\title{
Stage I Mycosis Fungoides AJCC v7
}

National Cancer Institute

\section{Source}

National Cancer Institute. Stage I Mycosis Fungoides A/CC v7. NCI Thesaurus. Code C7796.

Stage I includes: IA (T1, N0, M0, B0-1); IB (T2, N0, M0, B0-1). T1: Limited patches, papules, and/or plaques covering less than $10 \%$ of the skin surface. May further stratify into T1a (patch only) vs. T1b (plaque +/- patch). T2: Patches, papules, or plaques covering $10 \%$ or more of the skin surface. May further stratify into T2a (patch only) vs. T2b (plaque +/- patch). N0: No clinically abnormal peripheral lymph nodes; biopsy not required. M0: No visceral organ involvement. B0: Absence of significant blood involvement: $5 \%$ or less of peripheral blood lymphocytes are atypical (Sezary cells). B1: Low blood tumor burden: more than $5 \%$ of peripheral blood lymphocytes are atypical (Sezary cells) but does not meet the criteria of B2. (AJCC 7th ed.) 